\section{International Scientific Journal}

\section{Theoretical \& Applied Science}

p-ISSN: 2308-4944 (print) $\quad$ e-ISSN: 2409-0085 (online)

Year: 2017 Issue: $01 \quad$ Volume: 45

Published: $30.01 .2017 \quad \underline{\text { http://T-Science.org }}$

SECTION 6. Metallurgy and energy.
E.S. Belyaev

Candidate of technical Sciences, associate professor of «Materials science, technology of materials and heat treatment of metals») Nizhny Novgorod State Technical University n.a. R.E. Alekseev

E.V. Tumina Master student of the Department «Materials science, technology of materials and heat treatment of metals» Nizhny Novgorod State Technical University n.a. R.E. Alekseev

N. V. Makarov

Postgraduate student of the Department «Materials science, technology of materials and heat treatment of metals»)

Nizhny Novgorod State Technical University n.a. R.E. Alekseev

\title{
THE GEOMETRICAL CHARACTERISTICS OF THE HEAT AFFECTED ZONE IN THE LASER TREATMENT OF STEEL 38XH3MФA
}

Abstract: This paper examines the impact of the energy characteristics of the energy input (power density and speed of movement "laser spot" on the surface) in the laser surface treatment, and the initial state of the microstructure of steel 38XНЗМФА ГОСТ 4543-71 on the geometric dimensions of the heat-affected zone with the use of the method of mathematical planning of experiments.

The role of plasma processes in the laser treatment, the effect of the initial structural state of steel on the geometric dimensions of the heat-affected zone. The quantitative characteristics of these factors and their influence on the geometrical dimensions of the heat-affected zone.

Key words: geometric characteristics of the heat affected zone, laser processing, the processing mode, the power density, the speed of movement of the beam, mathematical planning experiment.

Language: Russian

Citation: Belyaev ES, Tumina EV, Makarov NV (2017) THE GEOMETRICAL CHARACTERISTICS OF THE HEAT AFFECTED ZONE IN THE LASER TREATMENT OF STEEL 38XH3MФA. ISJ Theoretical \& Applied Science, 01 (45): 153-159.

Soi: http://s-o-i.org/1.1/TAS-01-45-29 Doi: crostef https://dx.doi.org/10.15863/TAS.2017.01.45.29

\section{ГЕОМЕТРИЧЕСКИЕ ХАРАКТЕРИСТИКИ ЗОНЫ ТЕРМИЧЕСКОГО ВЛИЯНИЯ ПРИ ЛАЗЕРНОЙ ОБРАБОТКЕ СТАЛИ З8ХНЗМФА}

Аннотация: В настоящей работе рассматривается влияние энергетических характеристик энерговклада (плотность мощчноти и скорость перемещееня «лазерного пятна» по поверхности) при лазерной обработке поверхности и исходного состояния микроструктуры стали 38ХНЗМФА ГОСТ 4543 71 на геометрические размеры зоны термического влияния (ЗТВ) с применение метода математического планирования экспериментов.

Показана роль плазменных процессов при лазерной обработке, влияние исходного структурного состояния стали на геометрические размеры ЗТВ. Приведены количественные характеристики рассматриваемых факторов и их влияние на геометрические размеры ЗТВ.

Ключевые слова: геометрические характеристики зоны термического влияния, лазерная обработка, режим обработки, плотность мощности, скорость перемещения луча, математическое планирование эксперимента.

Введение. Поверхностная лазерная обработка применяется для повышения износостойкости поверхности изделий. В результате такой обработки на поверхности изделия образуется упрочненный слой с высокими значениями микротвердости. Для получения большей микротвердости на поверхности проводят предварительную объемную термообработку (закалка+отпуск). Обработка лазерным излучением производится 
локальным нагревом поверхности до сверхкритических температур и сверхбыстрым охлаждением нагретого участка за счет отвода тепла во внутренние слои материала. Упрочнение происходит на небольшую глубину материала. Величина глубины упрочнения влияет на работоспособность изделий в условиях износа. Целесообразно проводить обработку материала с режимами, обеспечивающими наибольшие размеры зоны термического влияния (ширину и глубину). Подбор таких режимов можно производить с помощью методов математического планирования экспериментов. Использование таких методов позволяет уменьшить число проводимых экспериментов и количественно оценить вклады каждого фактора в регрессионной модели. [1-5]

Объект исследования и оборудование. Исследования проводились на призматических образцах размерами 10х10х30 мм стали 38ХНЗМФА ГОСТ 4543 - 71, которая относится к улучшаемым. Химический состав стали 38ХНЗМФА приведен в [6,7].

Предварительно образцы подвергались объемной закалке с последующим отпуском при различных температурах. На каждой грани образцов получали «лазерные дорожки» различными режимами лазерной обработки. Обработка выполнена с помощью технологической установки «Латус-31», на базе оптического квантового генератора «Карат» с длиной волны лазерного излучения $\lambda=10,6$ мкм.

Разработка матрицы планирования эксперимента. При выполнении работы устанавливались зависимость ширины и глубины зоны термического влияния (ЗТВ) от плотности мощности лазерного излучения, скорости перемещения луча и структурного состояния материала (температуры отпуска) с помощью полного факторного эксперимента по планам $N=2^{K}$.

План $N=2^{K}$, где $K=3$ (число факторов), 2 число уровней (верхний +1 и нижний -1$), N=2^{3}=8$ - число опытов. Дополнительно с целью определения ошибки эксперимента проводилось четыре опыта на основном уровне. Для оценки кривизны поверхности отклика предусматриваем в матрице планирования экспериментов фиктивный фактор «Центр».

В качестве факторов влияющих на параметры отклика принимаем - плотность мощности $W$, скорость обработки $V$ и температура отпуска стали 38ХНЗМФА после закалки.

Основной уровень $x_{i}$ о имеет кодированное значение 0 . В натуральном масштабе факторы на основном уровне имеют следующие значения:

- Плотность мощности - 1,0·10 $10^{4} \mathrm{~B} / \mathrm{cm}^{2}$;

- Скорость обработки - 15 мм/сек;

- Температура отпуска $-400^{\circ} \mathrm{C}$.

Примем интервалы варьирования $\Delta x_{i}$ для плотности мощности, скорости обработки и температуры отпуска соответственно $0,2 \cdot 10^{4}$ $\mathrm{BT} / \mathrm{cm}^{2}, 5$ мм/сек и $200^{\circ} \mathrm{C}$. Верхний уровень имеет кодовое значение «+1», нижний - «-1». Факторы на верхнем уровне $x_{i} 0^{+} \Delta x_{i}$ в натуральном масштабе принимают следующие значения:

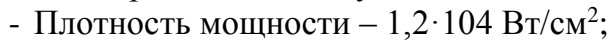

- Скорость обработки - 20 мм/сек;

- Температура отпуска $-600^{\circ} \mathrm{C}$.

На нижнем уровне $x_{i} 0^{-} \Delta x_{i}$ факторы в натуральном масштабе имеют значения:

- Плотность мощности - 0,8·104 Вт/см ${ }^{2}$

- Скорость обработки - 10 мм/сек

- Температура отпуска $-200^{\circ} \mathrm{C}$.

Матрица планирования эксперимента

Таблица 1

\begin{tabular}{|c|c|c|c|c|c|c|c|c|c|}
\hline \multirow{3}{*}{$\begin{array}{c}\text { № опыта } \\
i\end{array}$} & \multicolumn{7}{|c|}{ Значения факторов } & \multirow{3}{*}{$\begin{array}{c}\text { Ширина } b, \\
\text { мм }\end{array}$} & \multirow{3}{*}{$\begin{array}{c}\text { Глубина } h, \\
\text { мм }\end{array}$} \\
\hline & \multirow{2}{*}{$\begin{array}{c}x_{u} \\
\text { код. }\end{array}$} & \multicolumn{2}{|c|}{$x_{1}$} & \multicolumn{2}{|c|}{$x_{2}$} & \multicolumn{2}{|c|}{$x_{3}$} & & \\
\hline & & код. & нат., $\%$ & код. & нат., \% & код. & нат., $\%$ & & \\
\hline \multicolumn{10}{|c|}{ Опыты на верхнем и нижнем уровне } \\
\hline 1 & 1 & +1 & 1,2 & +1 & 20 & +1 & 600 & 2,82 & 0,30 \\
\hline 2 & 1 & -1 & 0,8 & +1 & 20 & +1 & 600 & 2,93 & 0,60 \\
\hline 3 & 1 & +1 & 1,2 & -1 & 10 & +1 & 600 & 2,99 & 0,70 \\
\hline 4 & 1 & -1 & 0,8 & -1 & 10 & +1 & 600 & 3,50 & 1,00 \\
\hline 5 & 1 & +1 & 1,2 & +1 & 20 & -1 & 200 & 3,07 & 0,48 \\
\hline 6 & 1 & -1 & 0,8 & +1 & 20 & -1 & 200 & 3,24 & 0,82 \\
\hline 7 & 1 & +1 & 1,2 & -1 & 10 & -1 & 200 & 3,37 & 0,87 \\
\hline 8 & 1 & -1 & 0,8 & -1 & 10 & -1 & 200 & 3,78 & 1,20 \\
\hline \multicolumn{10}{|c|}{ Параллельные опыты на основном уровне } \\
\hline $9-1$ & 0 & 0 & 1,0 & 0 & 15 & 0 & 400 & 3,25 & 0,74 \\
\hline $10-2$ & 0 & 0 & 1,0 & 0 & 15 & 0 & 400 & 3,55 & 0,72 \\
\hline $11-3$ & 0 & 0 & 1,0 & 0 & 15 & 0 & 400 & 3,30 & 0,73 \\
\hline $12-4$ & 0 & 0 & 1,0 & 0 & 15 & 0 & 400 & 3,37 & 0,75 \\
\hline
\end{tabular}

ISPC Perspectives in science for 2017, 


\begin{tabular}{l|lrl|l|ll} 
& ISRA (India) & $=\mathbf{1 . 3 4 4}$ & SIS (USA) & $=\mathbf{0 . 9 1 2}$ & ICV (Poland) & $=\mathbf{6 . 6 3 0}$ \\
Impact Factor: & ISI (Dubai, UAE) $=\mathbf{0 . 8 2 9}$ & PUHU (Russia) $=\mathbf{0 . 2 3 4}$ & PIF (India) & $=\mathbf{1 . 9 4 0}$ \\
& GIF (Australia) & $\mathbf{0 . 5 6 4}$ & ESJI (KZ) & $=\mathbf{1 . 0 4 2}$ & IBI (India) & $\mathbf{4 . 2 6 0}$
\end{tabular}

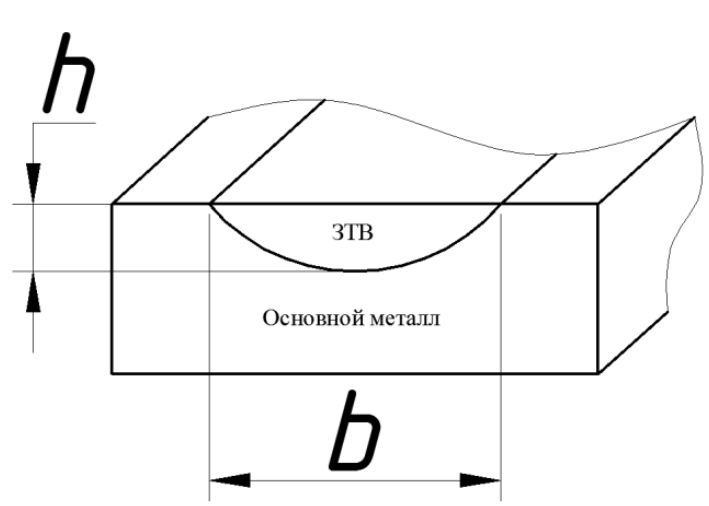

a)

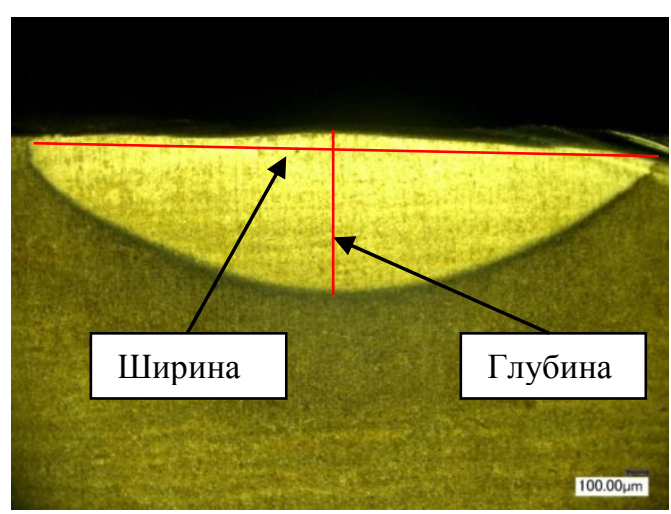

б)

$a$ - схема измерения глубины $(h)$ и ширины $(b)$ зоны термического влияния, $\sigma$ - внешний вид зоны термического влияния (после травления), х 100

Рисунок 1 - Схема измерений и внешний вид зоны термического влияния.

Матрица планирования экспериментов в кодированном и натуральном масштабах приведена в таблице 1. Так же в таблице 1 показаны параметры оптимизации - значения ширины $(b)$ и глубины $(h)$ 3ТВ.

Полученные данные и их обработка. Измерения ширины $(b)$ и глубины $(h)$ зоны термического влияния производились на подготовленном шлифе с помощью цифрового микроскопа Keyence $V H X-1000$ в соответствии со схемой, представленной на рисунке 1.

По полученным данным таблицы 1 проведен анализ с целью получения регрессионной модели для ширины $(b)$ и глубины $(h)$ зоны термического влияния соответственно. По результатам расчетов статистически незначимыми коэффициентами являются парные взаимодействия факторов, а также фиктивный коэффициент «Центр», что характеризует данную математическую модель как линейную.

Уравнения регрессии для ширины $(b)$, мм и глубины $(h)$, мм зоны термического влияния имеют вид соответственно:

$$
\begin{aligned}
& y_{1}=3,26-0,15 x_{1}-0,1975 x_{2}-0,1525 x_{3} \\
& y_{2}=0,74-0,159 x_{1}-0,196 x_{2}-0,096 x_{3}
\end{aligned}
$$

Гипотеза об адекватности полученных математических моделей по критерию Фишера при $\alpha=0,05$ не отвергается. Коэффициент детерминации полученных моделей $R^{2}$ составляет 0,72 и 0,99 соответственно. Уравнение, описывающее ширину ЗТВ, имеет достаточно низкий коэффициент детерминации.

Из уравнений 1 и 2 видно, что на ширину и глубину ванны лазерного упрочнения влияют плотность мощности, скорость обработки и температура предварительного отпуска. Bсе регрессионные коэффициенты имеют отрицательный знак - это значит, что увеличение каждого фактора уменьшает ширину и глубину 3ТВ. На ширину ванны плотность мощности и температура отпуска влияют приблизительно в равной степени. Влияние изменения температуры отпуска на глубину на порядок меньше, чем плотности мощности. Рост скорости обработки является основным фактором уменьшающим ширину и глубину 3ТВ.

Коэффициенты регрессии $b_{1}, b_{2}$ и $b_{3}$ уравнений 1 и 2 близки по значениям, следовательно, можно сделать вывод об одинаковом воздействии параметров варьирования, как на ширину, так и на глубину 3TB.

Карта Парето стандартизированных эффектов наглядно демонстрирует влияние каждого фактора на ширину и глубину ЗТВ лазерного излучения (рисунок 2,3$)$. 


\begin{tabular}{l|lrl|l|ll} 
& ISRA (India) & $=\mathbf{1 . 3 4 4}$ & SIS (USA) & $=\mathbf{0 . 9 1 2}$ & ICV (Poland) & $=\mathbf{6 . 6 3 0}$ \\
Impact Factor: & ISI (Dubai, UAE) $=\mathbf{0 . 8 2 9}$ & PUHU (Russia) $=\mathbf{0 . 2 3 4}$ & PIF (India) & $=\mathbf{1 . 9 4 0}$ \\
& GIF (Australia) & $\mathbf{0 . 5 6 4}$ & ESJI (KZ) & $=\mathbf{1 . 0 4 2}$ & IBI (India) & $\mathbf{4 . 2 6 0}$
\end{tabular}

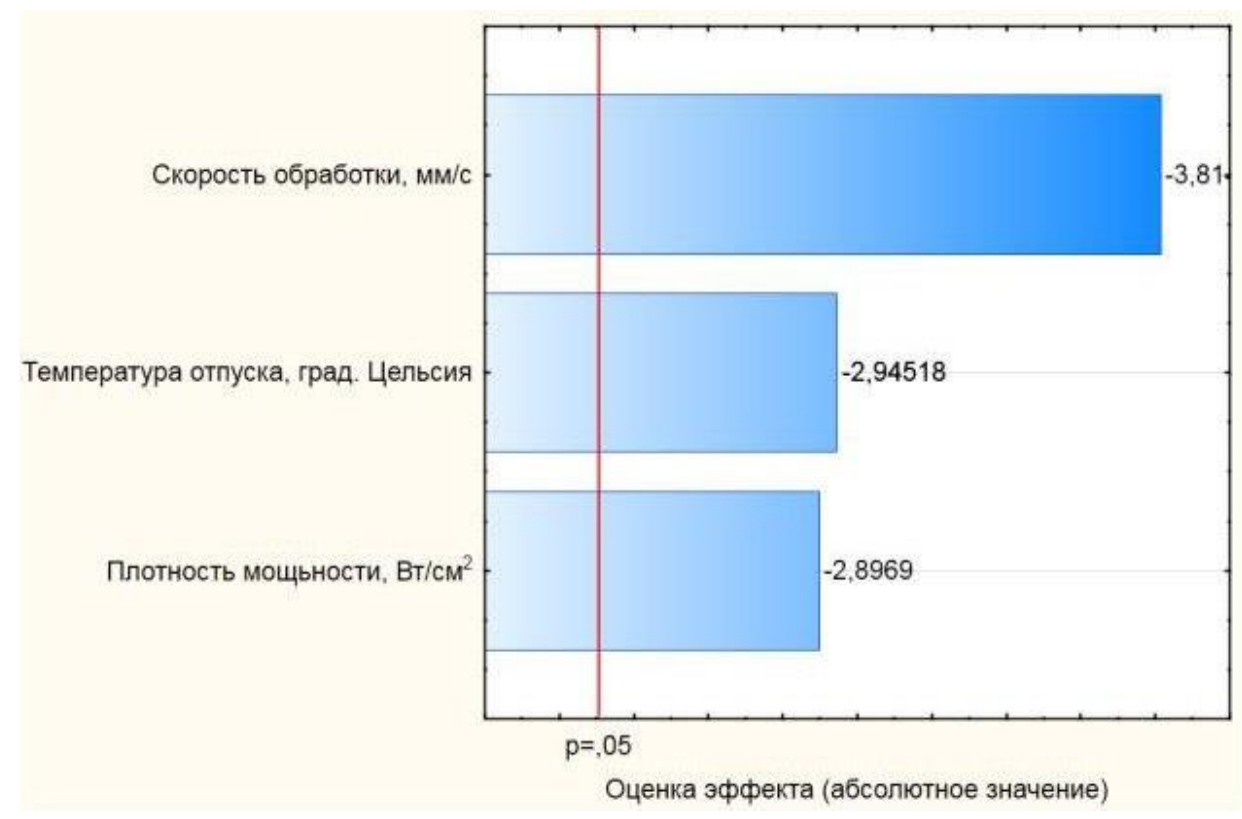

Рисунок 2 - Карта Парето стандартизированных эффектов для ширины ЗТВ

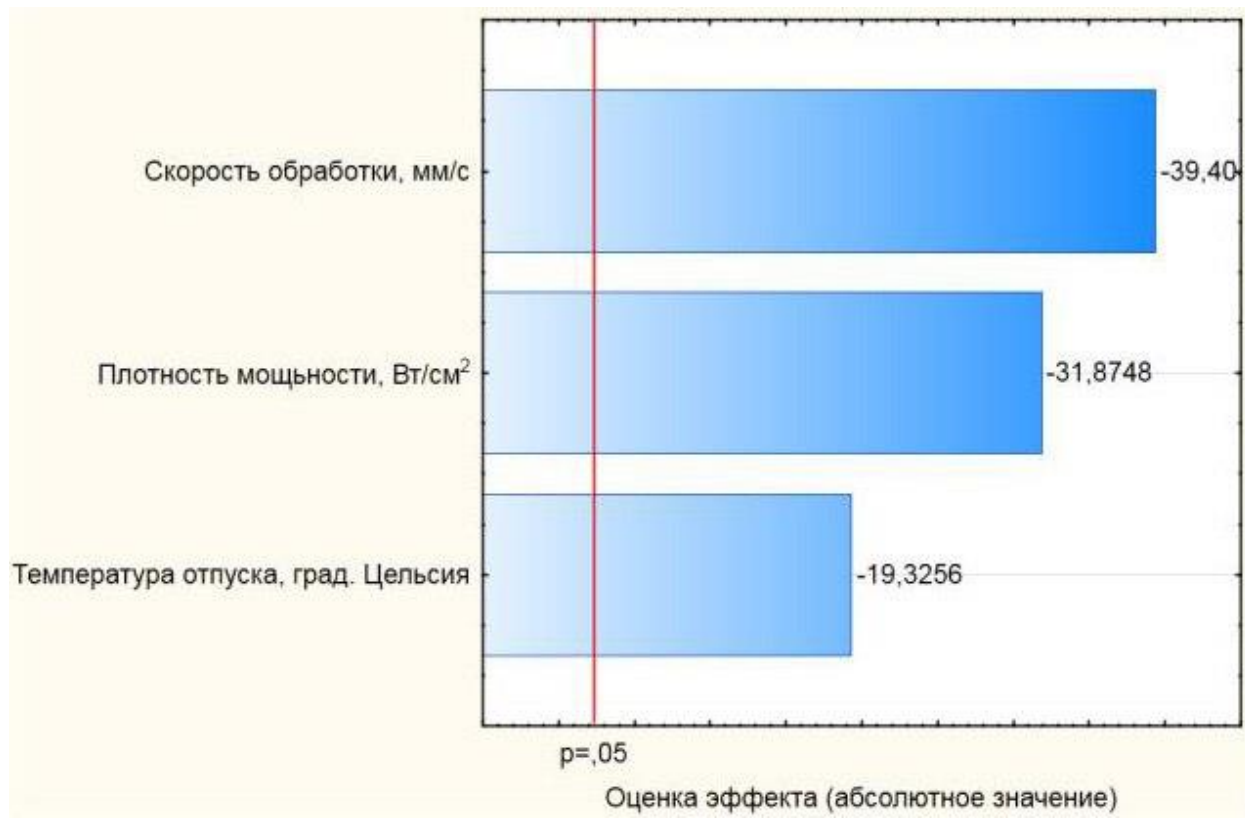

Рисунок 3 - Карта Парето стандартизированных эффектов для глубины ЗТВ

Плотность мощности является отношением мощности излучения к площади «лазерного пятна» на обрабатываемой поверхности. Согласно [3], в режиме обработки с оплавлением уменьшение плотности мощности в результате увеличения размера пятна и с уменьшением скорости перемещения лазерного луча позволяет резко повысить значения геометрических размеров ЗТВ. Полученные уравнения регрессии 1 и 2 показывают, что в выбранном интервале значений факторов все парные взаимодействия является статистически не значимыми.
Следовательно, взаимодействие факторов не влияет на ширину и глубину ЗТВ.

Обсуждение полученных результатов.

При разработке регрессионных моделей коэффициенты $b_{1}$, согласно опытным данным и расчету, имеют отрицательный знак. Очевидно, в выбранном интервале варьирования при лазерной обработке на воздухе с ростом плотности мощности у поверхности материала образуется «плазменное облако», приводящее к большому рассеиванию луча, тем самым снижая эффективную мощность излучения и

ISPC Perspectives in science for 2017, 


\begin{tabular}{|c|c|c|c|c|c|c|}
\hline Impact Factor: & $\begin{array}{l}\text { ISRA (India) } \\
\text { ISI (Dubai, UAF } \\
\text { GIF (Australia) } \\
\text { JIF }\end{array}$ & $\begin{array}{l}=1.344 \\
=0.829 \\
=0.564 \\
=1.500\end{array}$ & $\begin{array}{l}\text { SIS (USA) } \\
\text { PИНЦ (Russia } \\
\text { ESJI (KZ) } \\
\text { SJIF (Morocco }\end{array}$ & $\begin{array}{l}=\mathbf{0 . 9 1 2} \\
=\mathbf{0 . 2 3 4} \\
=\mathbf{1 . 0 4 2} \\
=\mathbf{2 . 0 3 1}\end{array}$ & $\begin{array}{l}\text { ICV (Poland) } \\
\text { PIF (India) } \\
\text { IBI (India) }\end{array}$ & $\begin{array}{l}=6.630 \\
=1.940 \\
=4.260\end{array}$ \\
\hline
\end{tabular}

сосредоточенность концентрации потока энергии на локальном участке [3]. При снижении значений плотности мощности ниже $0,8 \cdot 10^{4}$ $\mathrm{BT} / \mathrm{cm}^{2}$ вероятно уменьшение ширины и глубины ЗТВ за счет меньшего энерговклада несмотря на уменьшение интенсивности плазменных процессов на обрабатываемой поверхности. С целью исключения влияния «плазменного факела» необходимо принимать соответствующие меры [3].

Отрицательное значение коэффициентов $b_{2}$ в полученных регрессионных уравнениях согласуется с ранее проведенными исследованиями. Уменьшение скорости перемещения «лазерного пятна» по поверхности увеличивает время обработки, т.е. время пребывания площади поверхности материала, равную диаметру луча, под действием излучения:

$$
\tau=d / v
$$

где $\tau$ - время обработки, с;

$d$ - диаметр «лазерного пятна» на обрабатываемой поверхности, мм; $v$ - скорость перемещения лазерного пятна по поверхности, мм/с.

Структурное состояние стали 38ХНЗМФА согласно регрессионным уравнениям 1,2 снижает глубину и ширину ЗТВ, о чем свидетельствует отрицательный знак коэффициентов $b_{3}$ в соответствующих уравнениях. Образцы стали 38ХНЗМФА после закалки и отпуске при $t_{\text {omn }}=200^{\circ} \mathrm{C}$ образуют структуру бейнита. Средний отпуск при $t_{\text {oтn }}=400^{\circ} \mathrm{C}$ приводит к началу распада бейнита, а увеличение $t_{\text {отn }}$ до $600^{\circ} \mathrm{C}$ к образованию ферритно-карбидной смеси [4, 8-9]. Рост структурной неоднородности снижает теплопроводность стали [10], т.е. выделившиеся карбиды снижают скорость распространения теплоты от точечного источника излучения во внутренние слои материала. В результате наличия карбидной фазы и изменению ее размеров с увеличение температуры отпуска уменьшается глубина и ширина ЗТВ.

На рисунках 4,5 приводятся типичные графики зависимости плотности мощности и скорости обработки на ширину и глубину ЗТВ соответственно.

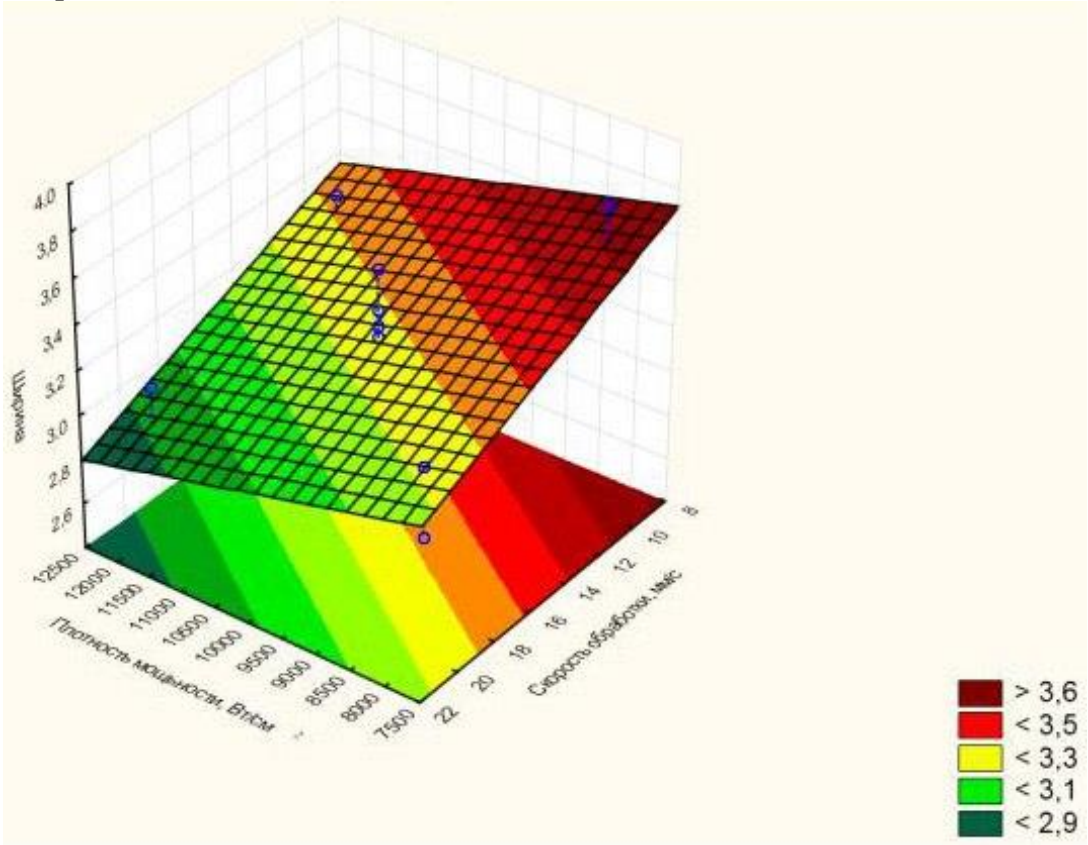

Рисунок 4 - Изменение ширина ЗТВ в зависимости от плотности мощности и скорости обработки при $t_{\text {omn }}=400^{\circ} \mathrm{C}$.

ISPC Perspectives in science for 2017, 


\begin{tabular}{l|lrl|l|ll} 
& ISRA (India) & $=\mathbf{1 . 3 4 4}$ & SIS (USA) & $=\mathbf{0 . 9 1 2}$ & ICV (Poland) & $=\mathbf{6 . 6 3 0}$ \\
Impact Factor: & ISI (Dubai, UAE) $=\mathbf{0 . 8 2 9}$ & PUHU (Russia) $=\mathbf{0 . 2 3 4}$ & PIF (India) & $=\mathbf{1 . 9 4 0}$ \\
& GIF (Australia) & $\mathbf{0 . 5 6 4}$ & ESJI (KZ) & $=\mathbf{1 . 0 4 2}$ & IBI (India) & $\mathbf{4 . 2 6 0}$
\end{tabular}

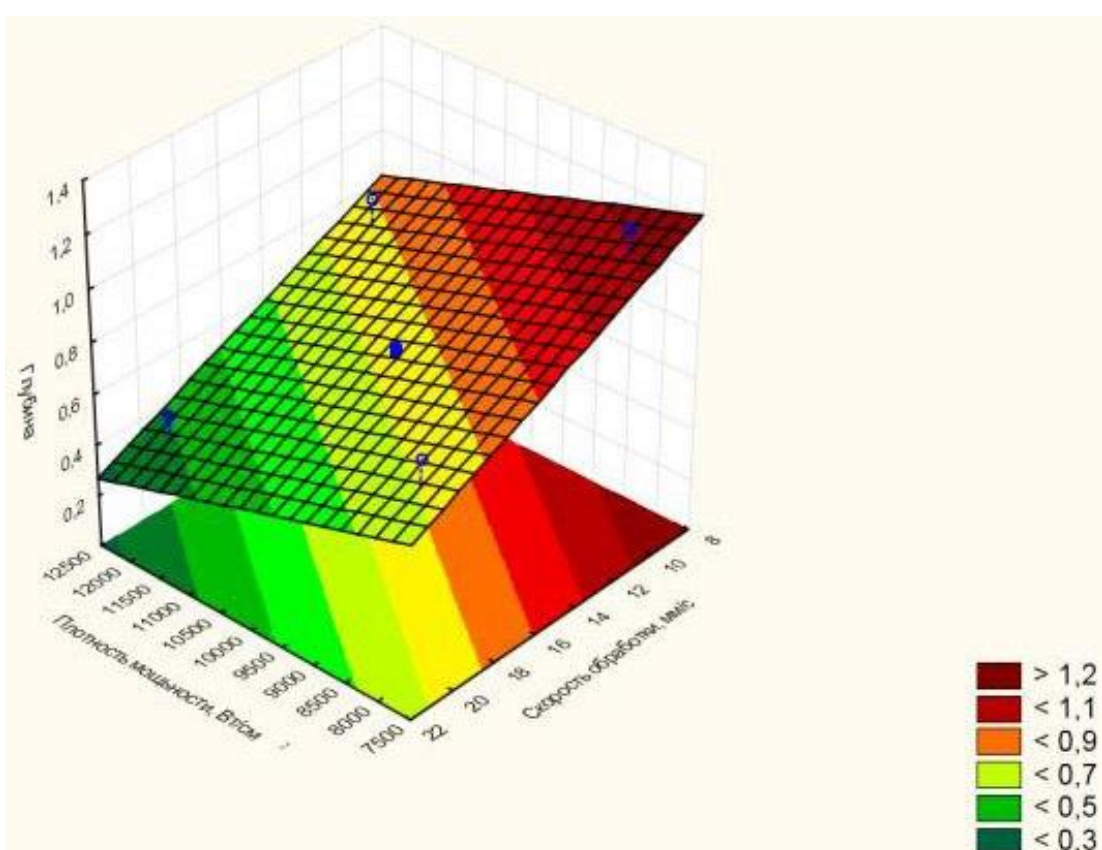

Рисунок 5 - Изменение глубины ЗТВ в зависимости от плотности мощности и скорости обработки при $t_{o m n}=400{ }^{\circ} \mathrm{C}$.

\section{Выводы.}

1. На ширину и глубину ЗТВ в равной степени влияют плотность мощности излучения, скорость обработки и структурное состояние материала (температура отпуска) исходя из близких значений коэффициентов $b_{1}, b_{2}$ и $b_{3}$ в разработанных регрессионных уравнениях 1 и 2 .

2. Коэффициенты $b_{1}, b_{2}$ и $b_{3}$ имеют отрицательный знак, что свидетельствует об обратнопропорциональной зависимости между каждым фактором и откликами - шириной и глубиной ЗТВ.

3. Увеличение плотности мощности лазерного излучения в рассмотренном интервале варьирования уменьшает геометрические размеры ЗТВ, что свидетельствует о развитии на поверхности обрабатываемого материала плазменных процессов приводящих к снижению теплового вклада в поверхность обрабатываемой стали.

4. Наибольшее влияние на геометрические размеры ЗТВ оказывает скорость лазерной обработки. Снижение скорости обработки увеличивает время пребывания площади поверхности материала, равную диаметру «лазерного пятна», под действием точечного источника теплоты, что ведет к росту размеров 3TB.

5. Повышение температуры предварительного отпуска к выделению карбидной фазы в структуре стали 38ХНЗМФА, что увеличивает структурную неоднородность и снижает теплопроводность стали, что ведет к снижению размеров ЗТВ

\section{References:}

1. Gavrilov GN (2011) Osobennosti formirovaniya struktury stalej pri lazernom termicheskom cikle [Tekst]/ Gavrilov G.N., Kastro V.A., Brauehr I.B., Belyaev E.S.//Zagotovitel'nye proizvodstva v mashinostroenii.- 2011.-№12.-p.38-41.

2. Kostromin SV (2013) Lazernaya zakalka rezhushchih kromok nozhej dlya obrabotki drevesiny [Tekst]/ Kostromin S.V., Belyaev E.S.//Aktual'nye voprosy sovremennoj tekhniki i tekhnologii. Sbornik dokladov XIII-j
ISPC Perspectives in science for 2017, Philadelphia, USA
Mezhdunarodnoj nauchnoj konferencii.Lipeck,2013.-p.59-61.

3. Grigor'yanc AG, Shiganov IN, Misyurov AI (2006) Tekhnologicheskie processy lazernoj obrabotki: Ucheb. Posobie dlya vuzov/ Pod red. A.G.Grigor'yanca.-M.: Izd-vo MGTU im. N.EH.Baumana, 2006.-664p.

4. Belyaev ES (2016) Primenenie metoda matematicheskogo planirovaniya ehksperimenta pri izuchenii processa kompleksnoj termicheskoj obrabotki stali 38HN3MFA 


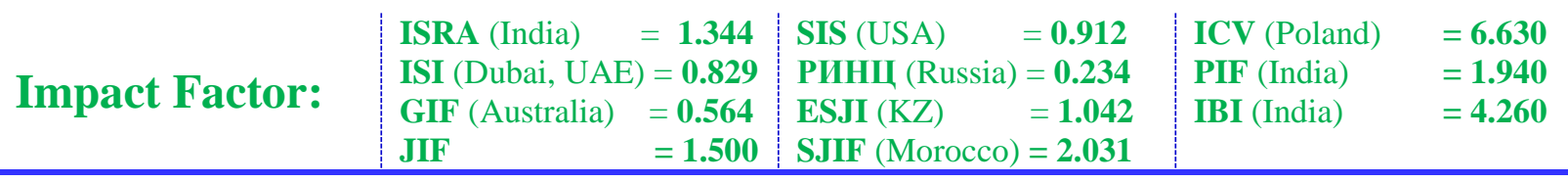

[Tekst]/ Belyaev E.S., Tumina E.V., Makarov N.V// Theoretical \& Applied Science. 2016. № 11 (43). p. 118-126

5. Belyaev ES (2016) Povyshenie iznosostojkosti rezhushchego instrumenta $\mathrm{s}$ plastinami iz tverdogo splava T15K6 [Tekst] / Belyaev E.S., Pavlov S.I., Svidenko E.V.// Uprochnyayushchie tekhnologii i pokrytiya. 2016. - № 3. - p. 6-9.

6. (1989) Marochnik stalej i splavov / V. G. Sorokin, A. V. Volosnikova, S. A. Vyatkin i dr; Pod obshch. red. V. G. Sorokina. - M.: Mashinostroenie, 1989. - $640 \mathrm{p}$.

7. (2003) Marochnik stalej i splavov. 2-e izd. dop. i ispr. / A. S. Zubchenko, M. M. Koloskov, YU.
V. Kashirskij i dr; Pod obshch. red. A. S. Zubchenko. - M.: Mashinostroenie, 2003. $784 \mathrm{p}$.

8. Bogoduhov SI (2015) Materialovedenie: uchebnik / S.I. Bogoduhov, E.S. Kozik. - Staryj Oskol: TNT, 2015. - 536 p.

9. Novikov II (1974) Teoriya termicheskoj obrabotki metallov. Uchebnik. 2-e izd. Novikov I.I. M., «Metallurgiya», 1974, 400 p.

10. (2011) Teploprovodnost' materialov: uchebnoe posobie / A.G. Korotkih; Tomskij politekhnicheskij universitet. - Tomsk: Izd-vo Tomskogo politekhnicheskogo universiteta, 2011. -97 p. 